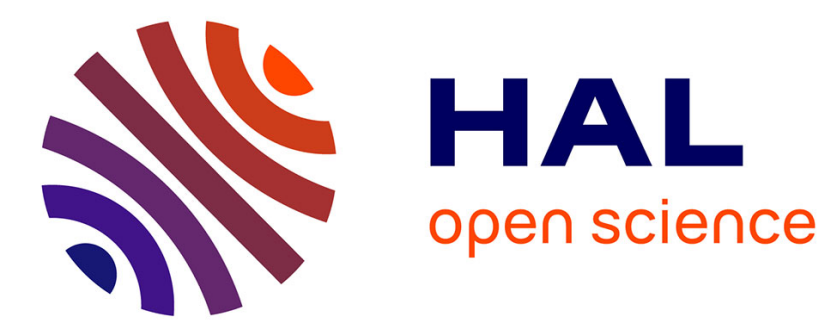

\title{
A deep-learning-based workflow to assess taxonomic affinity of hominid teeth with a test on discriminating Pongo and Homo upper molars
}

Zhixing Yi, Clément Zanolli, Wei Liao, Wei Wang

\section{- To cite this version:}

Zhixing Yi, Clément Zanolli, Wei Liao, Wei Wang. A deep-learning-based workflow to assess taxonomic affinity of hominid teeth with a test on discriminating Pongo and Homo upper molars. American Journal of Physical Anthropology, 2021. hal-03233436

\section{HAL Id: hal-03233436 \\ https://hal.science/hal-03233436}

Submitted on 24 May 2021

HAL is a multi-disciplinary open access archive for the deposit and dissemination of scientific research documents, whether they are published or not. The documents may come from teaching and research institutions in France or abroad, or from public or private research centers.
L'archive ouverte pluridisciplinaire HAL, est destinée au dépôt et à la diffusion de documents scientifiques de niveau recherche, publiés ou non, émanant des établissements d'enseignement et de recherche français ou étrangers, des laboratoires publics ou privés. 


\title{
A deep-learning-based workflow to assess taxonomic affinity of hominid teeth with a test on discriminating Pongo and Homo upper molars
}

\author{
Zhixing Yi, Clément Zanolli, Wei Liao, Wei Wang
}

\begin{abstract}
Objectives

Convolutional neural network $(\mathrm{CNN})$ is a state-of-art deep learning (DL) method with superior performance in image classification. Here, a CNN-based workflow is proposed to discriminate hominid teeth. Our hope is that this method could help confirm otherwise questionable records of Homo from Pleistocene deposits where there is a standing risk of mis-attributing molars of Pongo to Homo.

Methods and materials

A two-step workflow was designed. The first step is converting the enamel-dentine junction (EDJ) into EDJ card, that is, a two-dimensional image conversion of the three-dimensional EDJ surface. In this step, researchers must carefully orient the teeth according to the cervical plane. The second step is training the CNN learner with labeled EDJ cards. A sample consisting of 53 fossil Pongo and 53 Homo (modern human and Neanderthal) was adopted to generate EDJ cards, which were then separated into training set $(n=84)$ and validation set $(n=22)$. To assess the feasibility of this workflow, a Pongo-Homo classifier was trained from the aforementioned EDJ card set, and then the classifier was used to predict the taxonomic affinities of six samples (test set) from von Koenigswald's Chinese Apothecary collection.

Results

Results show that EDJ cards in validation set are classified accurately by the CNN learner. More importantly, taxonomic predictions for six specimens in test set match well with the diagnosis results deduced from multiple lines of evidence, implying the great potential of CNN method.

Discussion

This workflow paves a way for future studies using CNN to address taxonomic complexity (e.g., distinguishing Pongo and Homo teeth from the Pleistocene of Asia). Further improvements include visual interpretation and extending the applicability to moderately worn teeth.
\end{abstract}

\section{Introduction}


Human evolution in Asia remains a highly debated topic, especially regarding the chronospatial dynamics and interactions of Late Pleistocene modern humans with other hominin groups (Bae et al., 2017). The paleoanthropological record is scarce and by definition gives a partial picture of the past. Teeth represent the best preserved and most abundant remains in the fossil record, and they are also diagnostic elements to assess taxonomic affinities of fossil hominids. Among nonmetric features, the manifestations of tooth apparatuses such as cingulum, crista obliqua, cusp 5 , post-paracone tubercle, twinned paracone dentine horn, Carabelli's cusp, protoconule, midtrigonid crest give indications on taxonomic affinities (e.g., Filoux \& Wattanapituksakul, 2019; Ibrahim et al., 2013; Liao et al., 2019; Martin et al., 2017; Martinón-Torres et al., 2012; Radovic et al., 2019; Skinner et al., 2008; Wang, 2009; Zhang \& Harrison, 2017). Qualitative traits outline the morphological form, allowing researchers to infer the possible taxonomic identity for a given sample. For specific taxa-like Gigantopithecus blacki, it is possible to recognize them based on a combination of qualitative traits, fossil provenance, and fossil age (Zhang \& Harrison, 2017). However, due to morphological convergence, crown size overlap and other factors complicating taxonomic identification (like occlusal wear and taphonomy affecting crown and root morphology), the discrimination of Asian fossil taxa such as Pongo, Meganthropus, and Homo solely based on qualitative traits is challenging (Smith et al., 2018; Smith, Olejniczak, et al., 2009; Zanolli et al., 2015, 2019). Quantitative assessments of size, shape, and dental ontogenesis thus represent additional evidence to support taxonomic attribution. These methods mainly include dental crown size (mesiodistal and buccolingual diameters; e.g., Bae et al., 2014; Yao et al., 2020), two-dimensional (2D) dentine horn height (e.g., Olejniczak, Smith, Wang, et al., 2008), relative cuspal area (e.g., Bailey, 2004), crown tissue proportions and 2D/threedimensional (3D) enamel thickness (e.g., Bayle et al., 2010; Kono, 2004; Lockey et al., 2020; Martin, 1985; Olejniczak, Smith, Feeney, et al., 2008), occlusal fingerprint analysis (e.g., Kullmer et al., 2009), geometric morphometric analysis (e.g., occlusal crown, enamel-dentine junction (EDJ), and pulp chamber; e.g., Zanolli et al., 2012; Zanolli et al., 2019; le Luyer et al., 2016), dental development analysis (e.g., daily secretion rate, long-period line periodicity, crown formation time; e.g., Smith et al., 2006; Smith, Olejniczak, et al., 2009; Smith, Harvati, et al., 2009; Smith et al., 2010, 2018; Smith, 2016). Among the dental structural features, the developmental interface between enamel and dentine called EDJ has been demonstrated to be a reliable taxonomic proxy to discriminate various hominid taxa even at the subspecific level (Skinner et al., 2008, 2009, 2010; Zanolli et al., 2012, 2015, 2019).

Whenever possible, it is preferable to combine both qualitative and quantitative evidences to recognize hominid teeth to improve the reliability of taxonomic assessment. Nevertheless, for some dental material with no contextual information on provenience, stratigraphy and 
chronology, like the fossil hominid teeth from von Koenigswald's Chinese Apothecary collection, consensus is not reached on their taxonomic statuses even if both nonmetric and metric characteristics are described in great details (Ciochon, 2009; Harrison et al., 2014; Pope, 1983; Simons, 1963; Smith et al., 2018; von Koenigswald, 1957; Wolpoff, 1982).

Recently, deep learning (DL) approaches showed remarkable progress in segmentation, detection, recognition, classification, scene understanding (Ahmed et al., 2019; Gu et al., 2018; Litjens et al., 2017), opening toward new and promising perspectives for applications to the paleoanthropological record. One of the most encouraging DL methods is convolutional neural network $(\mathrm{CNN})$, which has been proven an excellent method with high accuracy in classification task (Chen et al., 2016; Shin et al., 2016). For instance, in the field of dentistry, CNN was employed to automatically recognize tooth categories (incisor, canine, premolar, and molar) from dentition images (Li et al., 2019; Xu et al., 2019). Most recently, CNN was used to extract microfossils automatically from micro-computerized tomography (micro-CT) data of sedimentary rock (Carvalho et al., 2020). However, despite the promising potential of this method, to the best of our knowledge, $\mathrm{CNN}$ has not been adopted to assess taxonomic affinities of hominid teeth in previous contributions. We aim here at testing the application of the CNN method to taxonomic discrimination with EDJ as the input file.

\section{Materials and methods}

\subsection{Materials}

The sample adopted here for CNN training consists of fossil Pongo and Homo (including Neanderthals and modern humans) upper molars. All fossil Pongo teeth $(n=53)$ are original samples and they were excavated from different sites in Southern China, including Ganxian cave, Naxian cave, Ganqian cave, Yicun cave, Sanxieshan cave, Shanzu cave, and Yanli cave. All these caves are in Guangxi province, China. The orangutan specimens were recognized based on the morphology of occlusal enamel surface and EDJ, and they exhibit morphology that closely approximates that of extant orangutans and clearly differ from fossil and recent humans. Most Pongo teeth are scanned by a customized $\mu$-XCT scanner (housed at the Institute of Vertebrate Paleontology and Paleoanthropology, Chinese Academy of Sciences) and the remains are scanned by a TX225-Actis Micro-CT system (housed at the University Museum, the University of Tokyo). Regarding Homo teeth, most of them $(n=47)$ were downloaded from open-source database, including ESRF (2020), MorphoMuseuM Database (2020), NESPOS Database (2020), and Morphosource Database (2020). The remaining Homo teeth $(n=6)$ are original samples. More details of these Pongo and Homo samples are documented in Supplementary Material (SM) Table 1. Note that these 106 Pongo and Homo maxillary molars were only used to train CNN. 
In addition, six maxillary molars (test dataset in Table 1) that from von Koenigswald's Chinese Apothecary collection were adopted to assess the performance of the trained CNN learner. The micro-CT data of these teeth are available in ESRF database. We choose these samples to test due to the following reasons. First, for the teeth in von Koenigswald's collection, the sites of origin, stratigraphic context and associated information are lost. Besides, many teeth are worn on the outside, further complicating their taxonomic attributions while the underlying EDJ is wellpreserved. Since it is difficult to assess the taxonomic affinities for these specimens that have lost contextual information (e.g., von Koenigswald's collection), the potential of CNN method can be appreciated based on tests conducted on the reference sample and on the capacity to assess the taxonomic attribution of these test specimens. Another consideration is that Smith et al. (2018) have applied various methods, including 2D enamel thickness, morphometric analysis on 2D EDJ, long-period line periodicity, and crown formation time, to deduce the taxonomic affinities for the aforementioned six molars (test dataset in Table 1), and they concluded that these molars belong to Pongo or Homo. Nevertheless, their results showed large morphometric overlap between Homo and Pongo in most analyses, leaving some doubts regarding some taxonomic assessments. Here, we reassess these six maxillary molars with a DL approach, enabling a mutual validation between the CNN results and the conventional results by Smith et al. (2018).

TABLE 1. Training, validation, and test dataset

Dataset Hossil Pongo Ho

$\begin{array}{llll}\text { Provenance }^{a} & \text { No. samples } & \text { Provenance }^{b} & \text { No. samples }\end{array}$

$\begin{array}{lll}\text { Training } & \text { Yanli cave } & \text { Qafzeh, Israel } \\ \text { Naxian cave } & \text { Gurgy, France } \\ \text { Ganxian cave } & \text { Krapina, Croatia } \\ \text { Ganqian cave } & \text { La Quina, France }\end{array}$

Sanxieshan cave

$\begin{array}{llll}\text { Validation } & \text { Yanli cave } & 11 & \text { Qafzeh, Israel }\end{array}$ 


$\begin{array}{ll}\text { Dataset Hossil Pongo Homo } & \end{array}$

\begin{tabular}{|c|c|c|c|c|}
\hline & Provenance $^{a}$ & No. samples & Provenance $^{b}$ & No. samples \\
\hline & Yicun cave & & Gurgy, France & \\
\hline & Naxian cave & & Krapina, Croatia & \\
\hline & Shanzu cave & & La Quina, France & \\
\hline & \multicolumn{4}{|l|}{ Ganxian cave } \\
\hline & \multicolumn{4}{|l|}{ Ganqian cave } \\
\hline Test & \multicolumn{4}{|c|}{ CA673, CA796, CA799, CA771, CA772, CA770 } \\
\hline
\end{tabular}

- ${ }^{a}$ All these caves are in Guangxi province, China.

- ${ }^{\mathrm{b}}$ Note that the provenances of four Homo samples are uncertain and these samples are stored at Evolutionary Anthropology Department, Duke University, USA. Please refer to SM Table 1 for more details.

\subsection{Methods}

We designed a DL-based workflow (Figure 1). The workflow can be summarized into two steps. The first step is making EDJ card, which is a 2D image representing the 3D EDJ surface. In EDJ card, colormap stands for height distribution from EDJ surface to cervical plane. The details for the first step are described below.

1. Scan the specimens using micro-CT or synchrotron micro-CT. The voxel size of the resultant Pongo volume data ranges from 21.96 to $40.00 \mu \mathrm{m}$ and 17.93 to $50.00 \mu \mathrm{m}$ for Homo teeth. The volume data are then imported into image processing software (e.g., ImageJ, Avzio, Gragonfly, VG Studio, and Mimics), and segmented therein using watershed algorithm. If necessary, manual correction is carried out to correct minor defects.

2. Generate surface model from segmented images using a constrained smoothing algorithm with a kernel size ranging from two to three (Figure 1(a)).

3. Import the surface model into Geomagic Design X.2016 and separate the coronal dentine from the tooth roots (other CAD software like Rhinoceros can also be adopted for the same purpose). Specifically, a spline curve is digitized along the cervical line (black dash 
line in Figure 1(a)). And then, cervical plane (red font in Figure 1(a)) is produced by means of fitting with the spline curve (Benazzi et al., 2014; Tafforeau, 2004). The cervical plane is used to separate the coronal dentine from the tooth roots (Figure 1(b)). Afterward, move and rotate the EDJ until the following three conditions have been met. First, the center of the cervical plane is set at the origin point. Second, the direction from the paracone tip to the protocone tip is parallel to the $\mathrm{x}$-axis. Third, the normal direction of the cervical plane is parallel to the $\mathrm{z}$-axis. Once the origin, $\mathrm{x}$-axis, and $\mathrm{z}$-axis have been determined, the y-axis is also determined. In Geomagic, the "Align" module is then used to rotate and move the EDJ. In this module, if origin (the center of the cervical plane) and two axes (the strait line between the paracone and protocone dentine horns, and the line orthogonal to the cervical plane) have been determined, then the target can be moved and rotated automatically. In other words, moving and rotating the EDJ are not performed by mouse dragging but based on morphological features of the EDJ, including cervix plane, paracone tip, and protocone tip. The EDJ is then exported as point cloud file (e.g., asc file) where each point has a 3D coordinate.

4. Make EDJ card. At the moment, CNN methods are not very efficient at dealing with 3D surface (Muhazid et al., 2020), so that we need to convert the 3D EDJ surface into 2D map. This process is performed in Surfer 12.0. In detail, convert the point cloud into grid data using Kriging algorithm and then visualize the grid data via the "Color Relief" module (Figure 1(c)). The outcome is EDJ card. The colormap in EDJ card describes the height pattern from EDJ surface to cervical plane. To weaken the tooth size effect on CNN training, we adopted two different schemes to ensure EDJ cards have approximate equal size. In scheme-I, each EDJ card was rendered with its own height range from minimum to maximum. In this way, the colormap in EDJ card describes the relative height pattern and hence the EDJ card is scale-free in the sense of height. Afterward, we proportionally shrunken each EDJ card to make it inscribed by a consistent square (1478 $\times 1478$, pixel unit). The second step allows all EDJ cards have approximately equal buccolingual diameter or mesiodistal diameter. In our samples, buccolingual diameter is larger than mesiodistal diameter. As a result, EDJ cards are inscribed in the buccolingual direction and hence all EDJ cards have approximate equal buccolingual diameters. In scheme-II, we first did a Procrustes superimposition of the EDJ surfaces of all specimens using the "Align Surface" module in Avizo 8.0. After the alignments, we made a statistic of minimum and maximum height for all EDJ cards. Not surprisingly, all EDJ cards show a minimum height (at the cervix position) of $0 \mathrm{~mm}$. Homo specimen 277 has the maximum height $(9.75 \mathrm{~mm}$, at the dentine horn tip of paracone) compared to other samples. After that, all EDJ cards were rendered with a consistent height range (0$9.75 \mathrm{~mm}$ ). It is necessary to mention that in scheme-I, there is no need to align all specimens before making EDJ cards while in scheme-II, all specimens need to be aligned to each other before making EDJ cards. Besides, in both schemes, all left teeth were mirrored for homologous comparisons. 


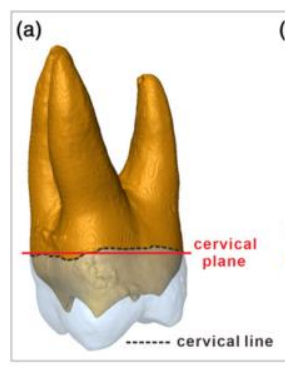

(c)

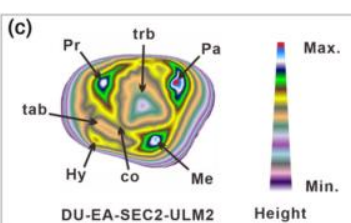

DU-EA-SEC2-ULM2 Height

Specimen/Prediction/Probability

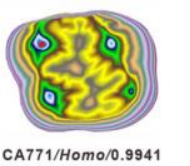

(b)

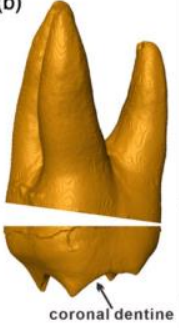

.

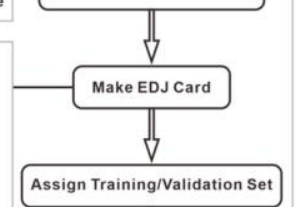

Assign Training/Validation Set Training CNN Learner

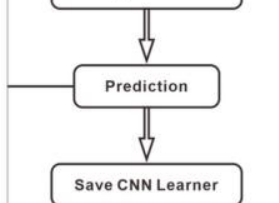

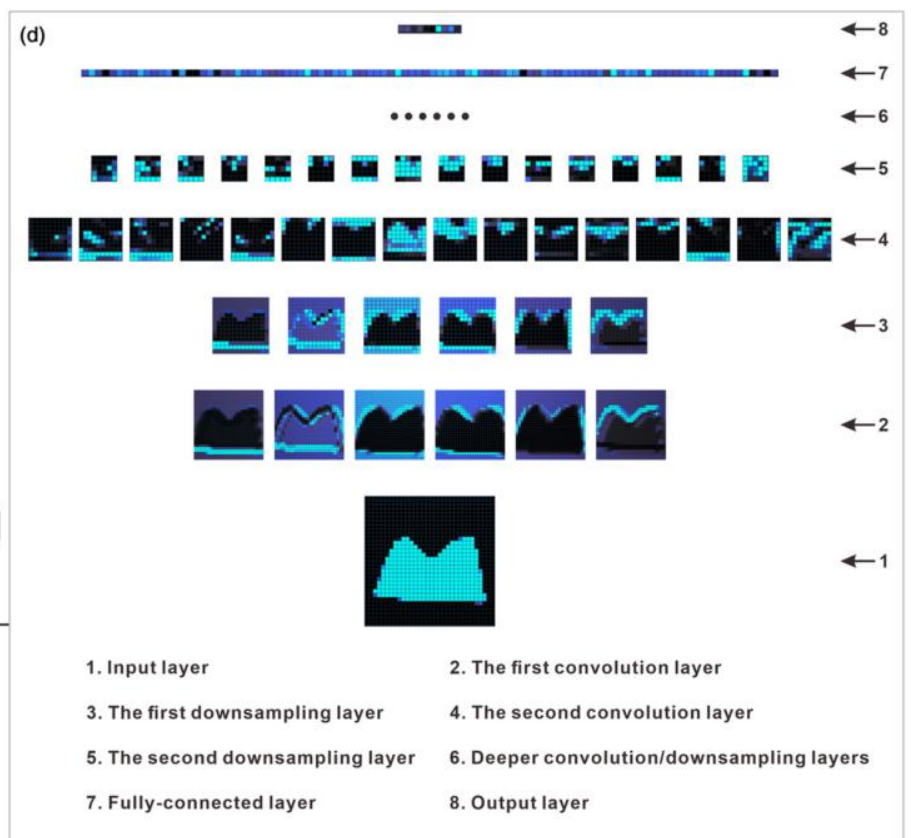

FIGURE 1. A deep learning (DL) -based workflow to classify hominid tooth. After microcomputerized tomography (micro-CT) image stacks have been segmented, (a) a virtual surface model is generated using constrained smoothing algorithm, and then (b) cervical plane is utilized to separate the coronal dentine from the tooth roots. (c) Visualize the coronal dentine by the "color relief" module in Surfer. Pr, protocone; Hy, hypocone; Pa, paracone; Me, metacone; co, crista obliqua; trb, trigon basin; tab, talon basin. After these processes, the three-dimensional (3D) enamel-dentine junction (EDJ) has been transformed to two-dimensional (2D) EDJ card. Repeat these processes to all samples until each one has its corresponding EDJ card. After that, EDJ cards are labeled with their taxa attributions and then assigned to training or validation dataset. When that is finished, (d) the convolutional neural network (CNN) learner can be employed to extract numerous features from EDJ card automatically (schematic figure was made from the website descripted in Harley (2015)). (e) The final trained CNN learner is adopted to predict taxonomic identity of EDJ card.

The above processes enable the transformation from 3D EDJ surface to 2D representation (EDJ card). After all samples have been processed and each sample has its corresponding EDJ card, the second step, training CNN, can be carried out by following the processes below.

1. Assign training/validation set. Most of the EDJ cards are assigned to the training set (e.g., $80 \%$ ) and the remaining cards to the validation set. To ensure that effective features will be extracted by CNN learner, the EDJ cards in training set need to cover as many different morphotypes as possible. Specifically, it is preferred that the trained samples are from different provenances and/or from different geologic time.

2. Train CNN learner. The brief principle of CNN is shown in Figure 1(d). Here and for example, we take a dentine section as the input layer (Layer 1). Matrix kernels are used to do convolution computation on the input layer and the outcome are convolution layer (Layer 2). Generally, macrolevel features (e.g., shape of the distal margin) will be extracted in the outer convolution layer (e.g., the first convolution layer). The convolution 
layer is followed by downsampling layer (Layer 3, also called as pooling layer), which is designed to reduce data size, reduce the phenomenon of over fitting, and improve fault tolerance. The commonly used schemes to obtain downsampling layer include max pooling and average pooling. The processes of convolution and downsampling are then iterated, and deeper layers are obtained (Layer 4, 5, and 6). The deeper the layer is, the more microscopic the feature is (e.g., pattern of the dentine horn tip). The fully connected layer (Layer 7) converts the feature matrixes of its previous layer into a vector, which contains discriminant information and determine classification result (output layer).

3. Give prediction. After the CNN learner has been trained and high accuracy has been reached in the validation set, the trained $\mathrm{CNN}$ learner can be employed to predict the identities of those EDJ cards, whose taxonomic affinities are uncertain (Figure 1(e)). If high accuracy is also reached in a considerable number of predictions, meaning that the trained model is robust, the learner can be saved for future use.

We coded a program using Jupyter notebook to perform the above processes and shared the program in Github: https:/github.com/CUG-YZX/Pongo-Homo-teeth-classifier. In the program, fastai (www.fast.ai) is employed as the basic application programming interface and ResNet18 (He et al., 2016; Howard \& Gugger, 2020) is invoked as the CNN implementation. The program is executable in Google Colaboratory (https://colab-research-google-com.proxy.mnhn.fr), where it is free to remotely access to high-performance computer.

To test how well the workflow is working, a sample consisting of 106 maxillary permanent first/second molars (53 fossil Pongo, 53 Homo [including modern human and Neanderthal]) were processed to generate their EDJ cards, which were further utilized to train and validate the CNN learner. Table 1 lists the brief information about training, validation, and test dataset. The detailed scheme of assigning training/validation dataset is provided in SM Table 1. It can be noted that both training and validation set have samples unearthed from different provenance. Thus, it is most likely that different morphotypes are assigned to training/validation set. This allocation improves the possibility that generalizable classification features will be acquired by CNN learner. It is necessary to mention that the process of CNN training do not involve the samples in test set. These samples are used to evaluate the performance of trained CNN learner.

\section{Results}

First, scheme-I was adopted to generate EDJ cards, which were then used to perform the DLworkflow and the results are shown in Figures 2-4-2-4 and Tables 2 and 3. The EDJ cards of Homo teeth are shown in Figure 2. These figures share some common characteristics. First, crista obliqua is evident in most cases (but see the exceptions like 248 and EH-BP9). Second, most samples display clear trigon basin and talon basin (but see the exceptions such as 252 and 289B). Third, hypocone is not higher than protocone in all cases except for KRD166. 

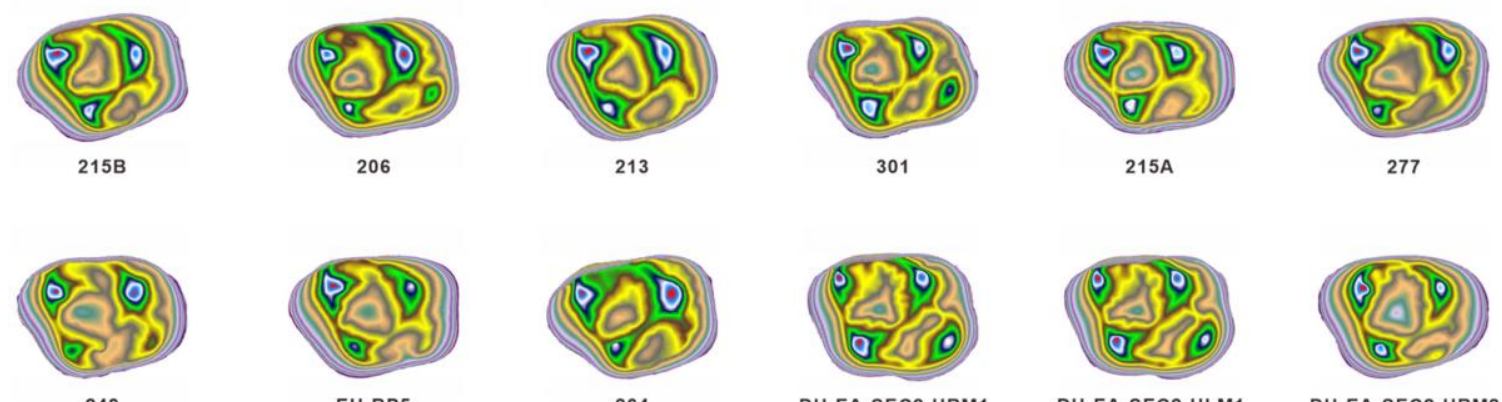

248

EH-BP5

DU-EA-SEC2-URM1

DU-EA-SEC2-ULM1

DU-EA-SEC2-URM2
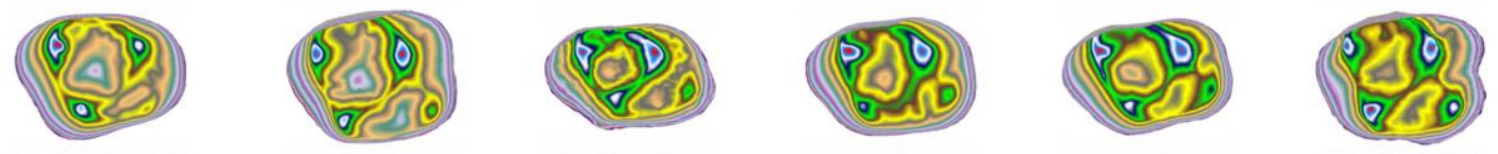

DU-EA-SEC2-ULM2

EH-UCL

EH-BP6-URM2

252
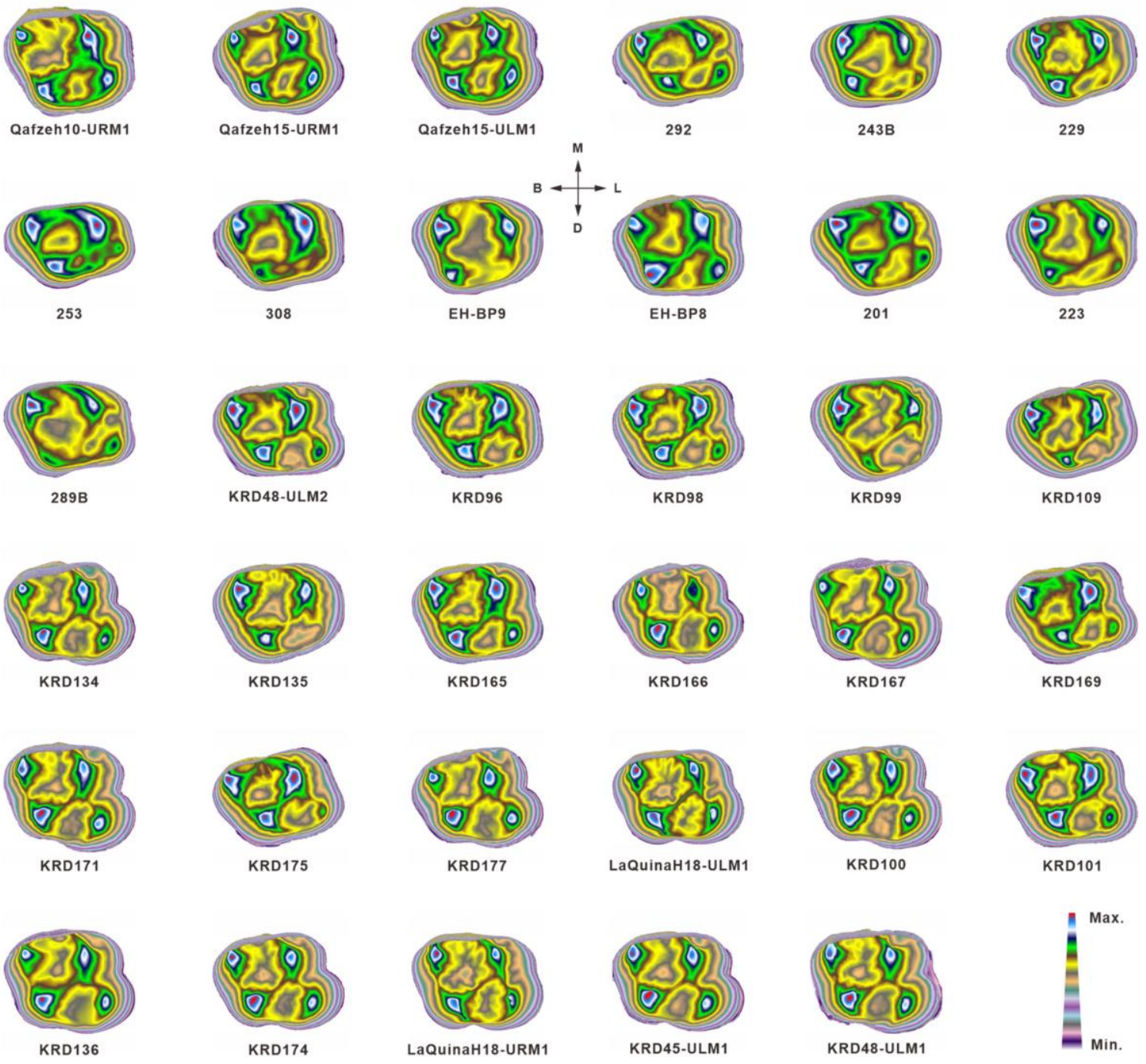

FIGURE 2. Enamel-dentine junction (EDJ) cards of studied Homo teeth. All left molars have been mirrored to the right position for comparison. Scheme-I was adopted to generate EDJ cards. 


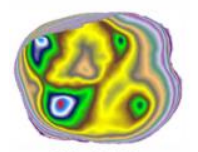

YL-15-22
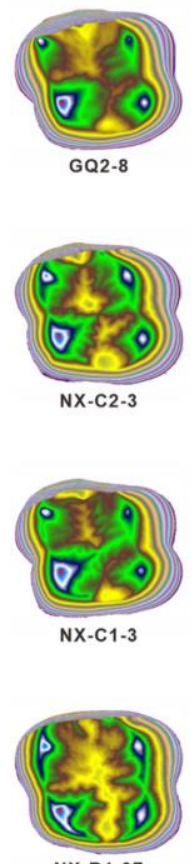

NX-B1-97

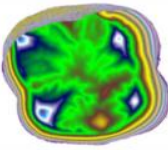

NX-B1-953
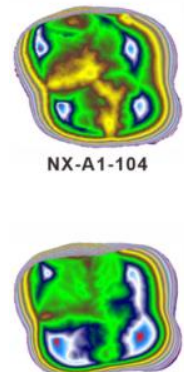

GQ2-6

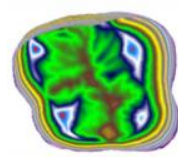

NX-A1-97
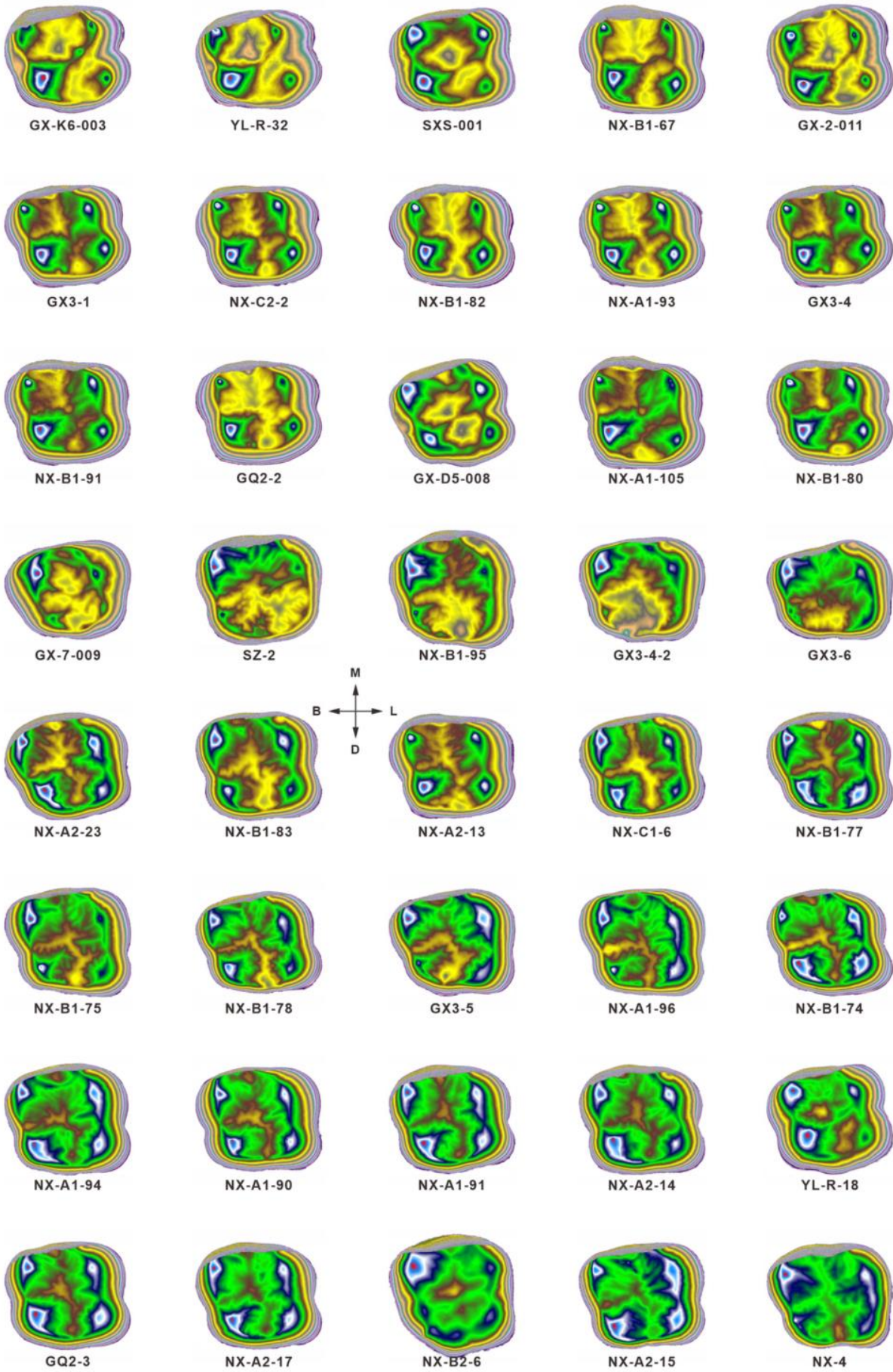

FIGURE 3. Ename-1-72
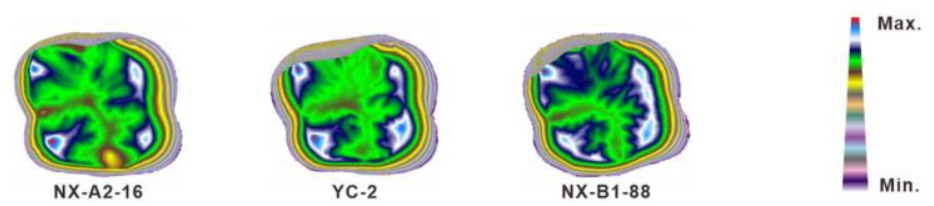
have been mirrored to the right position for comparison. Scheme-I. 
Prediction/Actual/Probability
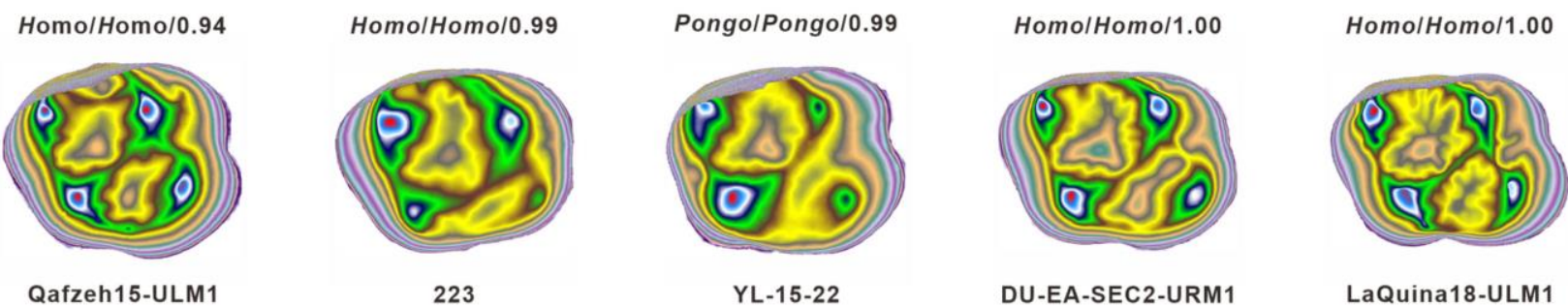

223

YL-15-22

DU-EA-SEC2-URM1

LaQuina18-ULM1

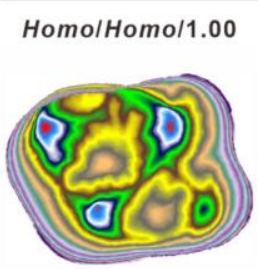

HomolHomol1.00

HomolHomol1.00

HomolHomol1.00

HomolHomo/1.00

KRD98
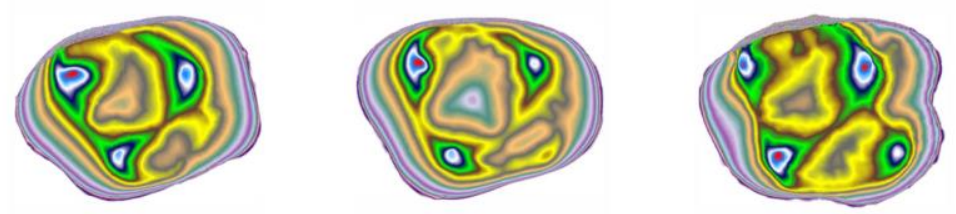

DU-EA-SEC2-ULM2

EH-BP6-URM1

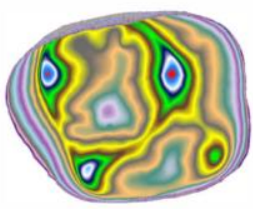

EH-UCL

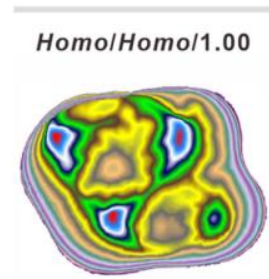

HomolHomol1.00

PongolPongo/1.00

PongolPongo/1.00

Pongo/Pongo/1.00

KRD101
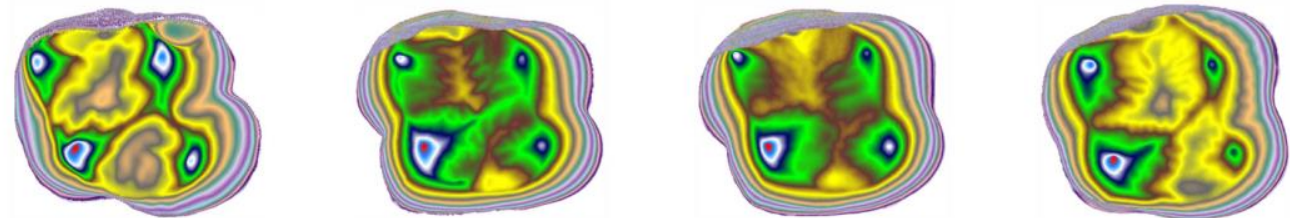

$$
\text { NX-C1-3 }
$$

GQ2-8

GX-2-011
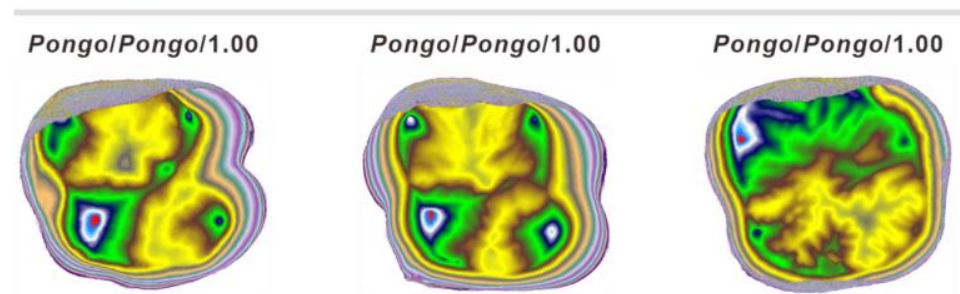

PongolPongo/1.00

PongolPongo/1.00

GX-K6-003

NX-B1-67

SZ-2
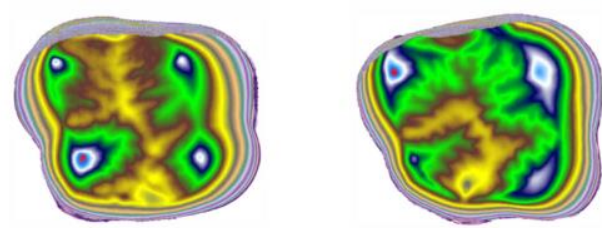

NX-A2-13

GX3-5

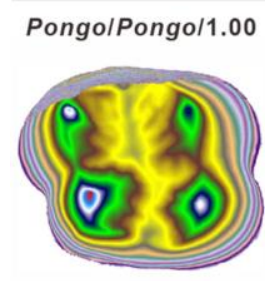

NX-B1-82
PongolPongo/1.00

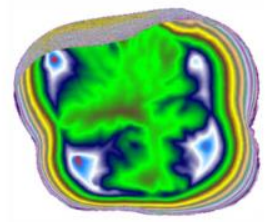

YC-2

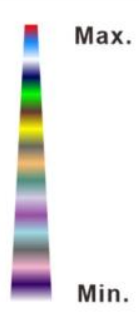

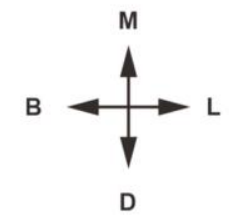

FIGURE 4. Predictions for the validation set. Scheme-I 
TABLE 2. Train loss, valid loss, and accuracy (in validation set) during training. Scheme-I

\begin{tabular}{llll}
\hline Training cycle & Train loss & Valid loss & Accuracy (\%) \\
\hline 1 & 0.3848 & 0.0523 & 100 \\
2 & 0.3344 & 0.0046 & 100 \\
\hline
\end{tabular}

TABLE 3. Taxonomic predictions for test set by present study and the corresponding diagnostic results provided by Smith et al. (2018) using other methods. Scheme-I

\begin{tabular}{|c|c|c|c|c|c|}
\hline \multirow[t]{2}{*}{ Specimens } & \multirow[t]{2}{*}{ EDJ card $^{a}$} & \multicolumn{2}{|c|}{ Present study } & \multicolumn{2}{|c|}{ Smith et al. (2018) } \\
\hline & & Prediction & Probability & Affinity & Methods $^{b}$ \\
\hline CA673 & & Pongo & 0.9329 & Pongo & $\mathrm{A}, \mathrm{B}, \mathrm{C}$ \\
\hline CA770 & & Homo & 0.9995 & Homo & $\mathrm{A}, \mathrm{B}, \mathrm{C}, \mathrm{D}$ \\
\hline CA771 & & Homo & 0.9941 & Homo & $\mathrm{A}, \mathrm{B}, \mathrm{C}$ \\
\hline CA772 & & Homo & 0.9995 & Homo & $\mathrm{C}$ \\
\hline CA796 & & Pongo & 0.9857 & Pongo & $\mathrm{A}, \mathrm{B}, \mathrm{C}, \mathrm{D}$ \\
\hline CA799 & & Pongo & 0.9999 & Pongo & $\mathrm{A}, \mathrm{B}$ \\
\hline
\end{tabular}

- Abbreviation: EDJ, enamel-dentine junction.

- ${ }^{\text {a }}$ Colormap is same as that shown in Figure 4. 
- $\quad$ b Methods applied by Smith et al. (2018): A-2D average enamel thickness, B-geometric morphometric analysis on 2D EDJ, C-long-period line periodicity, and D-crown formation time.

Unlike the Homo teeth where the crista obliqua is evident in most cases, this feature is modestly developed in a considerable number of Pongo cards (e.g., NX-B1-97, SZ-2, GQ2-3, and GQ2-2 in Figure 3). This finding was also previously observed by Zanolli et al. (2019). Due to that, trigon basin connects with talon basin and they together merge into a narrow basin in these cases such as NX-C1-6, NX-B1-97, NX-A1-97, NX-A2-23, GQ2-2, and GQ2-3. Besides, some Pongo cards show that the hypocone is higher than the protocone (e.g., NX-B1-67, GQ2-8, GQ2-6, NXB1-77, and YC-2).

The above analysis implies that discriminant information can be recorded on EDJ card. Although only some obvious differences have been pointed out in the above comparisons, it is highly likely that there exist other subtle taxonomic features, which are effective in classifying Pongo and Homo. It is laborious to find out these classification features in a manual way. Even if diagnostic features are found to be valid to classify Pongo and Homo, there is no guarantee that these features are efficient in classifying other hominid teeth. With CNN, distinguishing features can be detected automatically. In a specific classifying task, numerous features (including both diagnostic and nondiagnostic features) are first extracted in form of matrixes and these features are then analyzed by $\mathrm{CNN}$ to find out which ones are useful in the target task. The advantage of $\mathrm{CNN}$ is that it finds distinguishing features automatically and can learn to recognize more when fed with more specimens/samples.

Table 2 lists the training details. In the table, train loss and valid loss are quantitative parameters reflecting classification quality in training set and validation set, respectively. The lower the loss is, the higher the classification quality is. Valid loss is lower than train loss, meaning that there is a low probability of overfitting. Both train loss and valid loss are lower in the second training cycle than the first one, indicating that model performance has been improved during the second cycle. Since the prediction accuracy in validation set has reached $100 \%$ in the first two training cycles and valid loss is small, the training is basically accomplished, and more training cycles are not necessary.

The details of predictions for validation set are shown in Figure 4. All 22 samples have been classified accurately, indicating that effective features are recognized by CNN learner. It is not a surprise that such high accuracy is reached, because plentiful features on the EDJ card have been compared and with high probability, there exits some features possessing taxonomic value. In addition, there is high similarity between training set and validation set. In fact, most samples in 
validation set have at least one contemporaneous and same-provenance homologue in training set (SM Table 1).

Table 3 lists the predictions for test set. The samples in test set are not involved in the previous training process, meaning that CNN learner has not "seen" these samples before. With high predictive probabilities, predictions match well with the diagnostic results based on other routine methods (Table 3). This result strengthens the conclusion that generalizable distinguishing features of taxonomic value have been learned by $\mathrm{CNN}$.

When scheme-II is used to generate EDJ cards, then corresponding results are found in SM Figures 1-3 and SM Tables 2 and 3. SM Figure 3 shows that the samples in validation set were classified accurately and SM Table 3 also shows that the predictions by CNN learner match well with the diagnostic results deduced from previous studies (Smith et al., 2018). These results indicate that both scheme-I and scheme-II are compatible with the proposed DL workflow.

\section{Discussion}

During the Pleistocene, at least four hominid taxa, including Pongo, Gigantopithecus, Meganthropus, and Homo coexisted in Asia (Zanolli et al., 2019). Pongo and Homo teeth frequently co-occur in karstic Pleistocene sediments from Southern Asia (Wang et al., 2007). In addition to the spatio-temporal overlap between Pongo and Homo, they experienced a similar reduction trend of the dentition, associated with a convergence in molar morphology (Smith, Olejniczak, et al., 2009). Both orangutans and hominins have variably wrinkled molars with low cuspal relief and oval occlusal outline (Ortiz et al., 2019). In addition, molars of both taxa overlap for a number of quantitative variables, including enamel thickness, daily secretion rate, crown formation time, 2D geometric morphometric analyses of the EDJ (Martin, 1985; Olejniczak, Tafforeau, Feeney, \& Martin, 2008; Smith, 2016; Smith et al., 2011; Smith et al., 2018). The situation is further complicated by the relatively high frequency of upper and lower fourth molars (M4s) in Pongo (Bergstrom et al., 2016; Hooijer, 1948) and by the fact that most of the fossil hominid dental specimens are isolated teeth, with unsecure metameric attribution (Smith et al., 2018). On account of these similarities between Pongo and Homo molars, multiple lines of evidence are often necessary to deduce the taxonomic status of a given sample. Thus, an attempt of $\mathrm{CNN}$ was carried out here to test whether this method could distinguish Pongo/Homo molars. The results of this DL-based study on classifying Pongo and Homo upper molars are highly encouraging, with predictions that show high accuracy in both validation and test set. However, more importantly, the results are achieved on the conditions that sample size of training set is still small, and some human samples are even not from Asia. 
Like other statistical methods, the main issue with this Pongo-Homo classifier is that it needs sufficient representatives of both orangutans and hominins in the training set to give relevant results. A potential bias here is that the fossil Pongo specimens used for training are restricted to Guangxi province, China. Samples from other regions (e.g., Southeast Asia) are absent. Besides, the ages of these Pongo samples range from late Middle Pleistocene to Late Pleistocene, as indicated by a preliminary analysis on fauna assemblage. However, additional samples from other periods (e.g., Early Pleistocene) are not involved in the training. Since orangutan molars vary chronospatially, possibly due to the presence of the diversification of various species during the Pleistocene (Harrison et al., 2014; Ibrahim et al., 2013; Tshen, 2016; Wang et al., 2014), these training samples are unlikely to cover all of the past variation of Pongo molars. The situation of biased sampling is even worse for Homo. First, nearly all Homo samples are from Europe, whereas there is an acute shortage of local (Asia) representatives. Moreover, only two species $(H$. sapiens and Neanderthal) are included in the training without other hominins like H. erectus. Likewise, the human samples in training set are not expected to contain all diagnostic features of Homo molars.

Given the above reasons, it is better to take the current trained CNN learner as a trial version. The future inclusion of more specimens sampling broader chrono-geographic areas and including more taxa, like Gigantopithecus and Meganthropus, will enable strengthening the model and make it even more reliable than it is at the moment. In this prospect, open access to more microCT data of hominid teeth would help improving the model and building larger, more inclusive reference databases to increase the taxonomic scope. In principle, as both the number of specimens and the variability of morphotypes increase in training set, the model performance will improve. For these reasons and in order to facilitate the application of this DL CNN model for future studies, we share the program on Github, allowing other scholars to optimize this taxonomic classifier.

Future efforts need to be concentrated on the visual interpretation of CNN learner. Although plenty of studies have shown the superior performance of CNN in classification task, this model remains a black box to some extent, because the structure and algorithm of CNN are extremely complicated, and the end-to-end learning strategy is adopted therein (Lipton, 2018; Zhang \& Zhu, 2018). In other words, while prediction is accurate, it is difficult to locate which parts of the image are taken as the effective features in the trained CNN learner. For this reason, more and more DL coders devote their efforts to visualizing the CNN structure and find out the target informative features (Kim et al., 2019). To achieve this goal, multidisciplinary collaborations, especially with DL coders, will be required. 
A promising perspective would be to test whether the CNN learner can be extended to moderately worn teeth (with worn dentine horn tips). In this paper, nearly all samples are slightly worn but without dentine exposure and hence the EDJ cards are complete. However, a large part of the unearthed fossil hominid teeth exhibits moderate occlusal wear affecting some of the dentine horns. For these samples, the generated EDJ cards are incomplete. In this situation, EDJ card lose information on dentine horn tips and are not homologous. However, CNN can also be applied to partial EDJ card and if the features recorded on the remaining parts are enough to identify a specific taxon for a given sample, then it would be worth exploiting the potential of incomplete EDJ cards. For example, Ortiz et al. (2019) have demonstrated that the shape of EDJ mesial fovea of upper molar is a distinguishing feature that capable of separating Pongo from Homo. In this case, only partial information is needed, supporting the potential of incomplete EDJ card.

Another step forward will consist of developing further DL methods that can be applied to 3D structures. To this day, thanks the large availability of reference images necessary to build strong training models and the straightforward mathematical architecture behind, 2D DL CNN is successfully used for many applications in various fields, including now in paleoanthropology. Even if 3D objects are increasingly available and the computational power necessary to run complex models continuously increases, there are still many challenges to overcome to develop efficient DL architectures to extract and characterize features from 3D shapes made of irregular meshes or point clouds (Muhazid et al., 2020).

\section{Conclusion}

For the first time, a DL-based workflow is proposed here to assess taxonomic affinity of hominid teeth. The workflow comprises of two steps. The first step is a transformation from 3D EDJ surfaces to 2D EDJ cards. The second step is training CNN with the labeled EDJ cards. We coded a program to perform the second step and share it with the scientific community. Our first result shows that Pongo and Homo are accurately separated by CNN learner in validation set. And more encouragingly, predictions for the test set also match well with the results deduced from a combination of conventional methods, implying the great potential of CNN method.

\section{Acknowledgments}

For access to the micro-CT image stacks of hominid teeth, the authors thank the following institutions: Rockfeller Museum (Israel); Evolutionary Anthropology, Duke University (USA); Ostéothèque de Pessac, Université de Bordeaux (France); Musée d'Archéologie nationale 
(France); Croatian Natural History Museum (Croatia); International Centre for Theoretical Physics (Italy); University College of London (UK). For scanning and technical assistance, the authors thank Song Xing, Lei Pan, and Reiko T. Kono. The authors are indebted to Prof Shuifu Li (China University of Geosciences) for his help on EDJ card making. The authors sincerely appreciate the beneficial discussions with Prof Yongbiao Wang and Dr Tan Wang. The authors sincerely appreciate two anonymous reviewers for their constructive comments and suggestions. This study was supported by the funds from the National Natural Science Foundation of China (grant Nos. 40772011 and 41572023), and by a grant of the Bagui Scholar of Guangxi.

\section{Conflict of interest}

The authors declare no conflict of interest.

\section{Author contributions}

Zhixing Yi: Conceptualization; investigation; methodology; validation; writing-original draft; writing-review and editing. Clément Zanolli: Methodology; validation; writing-original draft; writing-review and editing. Wei Liao: Conceptualization; funding acquisition; investigation; methodology; writing-original draft; writing-review and editing. Wei Wang: Conceptualization; funding acquisition; investigation; methodology; validation; writing-original draft; writingreview and editing.

\section{Funding information}

National Natural Science Foundation of China, Grant/Award Numbers: 40772011, 41572023; Bagui Scholar of Guangxi

Ahmed, E., Saint, A., Shabayek, A., Cherenkova, K., Das, R., Gusev, G., \& Aouada, D. (2019) A survey on deep learning advances on different 3D data representations. arXiv, 1, $1808.01462 \mathrm{v} 2$.

Bae, C. J., Douka, K., \& Petraglia, M. D. (2017). On the origin of modern humans: Asian perspectives. Science, 358(6368), eaai9067. https://doi.org/10.1126/science.aai9067

Bae, C. J., Wang, W., Zhao, J. X., Huang, S. M., Tian, F., \& Shen, G. J. (2014). Modern human teeth from Late Pleistocene Luna Cave (Guangxi, China). Quaternary International, 354, 169-183. https://doi.org/10.1016/j.quaint.2014.06.051

Bailey, S. E. (2004). A morphometric analysis of maxillary molar crowns of Middle-Late Pleistocene hominins. Journal of Human Evolution, 47(3), 183-198. 
https://doi.org/10.1016/j.jhevol.2004.07.001

Bayle, P., Macchiarelli, R., Trinkaus, E., Duarte, C., Mazurier, A., \& Zilhao, J. (2010). Dental maturational sequence and dental tissue proportions in the early Upper Paleolithic child from Abrigo do Lagar Velho, Portugal. Proceedings of the National Academy of Sciences of the United States of America, 107(4), 1338-1342. https://doi.org/10.1073/pnas.0914202107

Benazzi, S., Panetta, D., Fornai, C., Toussaint, M., Gruppioni, G., \& Hublin, J. J. (2014). Technical Note: Guidelines for the Digital Computation of 2D and 3D Enamel Thickness in Hominoid Teeth. American Journal of Physical Anthropology, 153(2), 305-313. https://doi.org/10.1002/ajpa.22421

Bergstrom, K., Jenkins, K., Levy, J., Velasquez, R. J., Lewis, P. J., \& Campbell, T. L. (2016). Computed tomography survey of supernumerary molars in extant orangutans with implications for studies of the primate fossil record. Paper presented at the Paleoanthropology Society 2016 Meeting, Atlanta, GA.

Carvalho, L. E., Fauth, G., Baecker Fauth, S., Krahl, G., Moreira, A. C., Fernandes, C. P., \& von Wangenheim, A. (2020). Automated Microfossil Identification and Segmentation using a Deep Learning Approach. Marine Micropaleontology, 158, 101890. https://doi.org/10.1016/j.marmicro.2020.101890

Chen, Y. S., Jiang, H. L., Li, C. Y., Jia, X. P., \& Ghamisi, P. (2016). Deep Feature Extraction and Classification of Hyperspectral Images Based on Convolutional Neural Networks. IEEE Transactions on Geoscience and Remote Sensing, 54(10), 6232-6251. https://doi.org/10.1109/tgrs.2016.2584107

Ciochon, R. L. (2009). The mystery ape of Pleistocene Asia. Nature, 459(7249), 910-911. https://doi.org/10.1038/459910a

ESRF Paleontological Microtomographic Database (2020). Retrieved from http://paleo.esrf.eu

Filoux, A., \& Wattanapituksakul, A. (2019). The Late Pleistocene Orangutan from Tham Prakai Phet: New discoveries. Annales De Paleontologie, 105(4), 287-293. https://doi.org/10.1016/j.annpal.2019.03.004

Gu, J. X., Wang, Z. H., Kuen, J., Ma, L. Y., Shahroudy, A., Shuai, B., . . Chen, T. (2018). Recent advances in convolutional neural networks. Pattern Recognition, 77, 354-377. https://doi.org/10.1016/j.patcog.2017.10.013

Harrison, T., Jin, C. Z., Zhang, Y. Q., Wang, Y., \& Zhu, M. (2014). Fossil Pongo from the Early Pleistocene Gigantopithecus fauna of Chongzuo, Guangxi, southern China. Quaternary International, 354, 59-67. https://doi.org/10.1016/j.quaint.2014.01.013

Harley, A. W. (2015). An Interactive Node-Link Visualization of Convolutional Neural Networks. In G. Bebis et al. (Eds.), International Symposium Visual Computing (pp. 867-877). Switzerland: Springer International Publishing.

He, K. M., Zhang, X. Y., Ren, S. Q., \& Sun, J. (2016). Deep Residual Learning for Image Recognition. Paper presented at the 2016 Ieee Conference on Computer Vision and Pattern Recognition, New York, USA.

Hooijer, D. A. (1948). Prehistoric teeth of man and of the orang-utan from Central Sumatra, with notes on the fossil orang-utan from Java and southern China. Zoologische Mededelingen, 29, 175-301.

Howard, J., \& Gugger, S. (2020). Fastai: A layered API for deep learning. Information, 11(2), 108. https://doi.org/10.3390/info11020108

Ibrahim, Y. K., Tshen, L. T., Westaway, K. E., Cranbrook, E. O., Humphrey, L., Muhammad, R. F., . . Peng, L. C. (2013). First discovery of Pleistocene orangutan (Pongo sp.) fossils in 
Peninsular Malaysia: Biogeographic and paleoenvironmental implications. Journal of Human Evolution, 65(6), 770-797. https://doi.org/10.1016/j.jhevol.2013.09.005

Kim, I., Rajaraman, S., \& Antani, S. (2019). Visual Interpretation of Convolutional Neural Network Predictions in Classifying Medical Image Modalities. Diagnostics, 9(2), 15. https://doi.org/10.3390/diagnostics9020038

Kono, R. T. (2004). Molar enamel thickness and distribution patterns in extant great apes and humans: new insights based on a 3-dimensional whole crown perspective. Anthropological Science, 112(2), 121-146. https://doi.org/10.1537/ase.03106

Kullmer, O., Benazzi, S., Fiorenza, L., Schulz, D., Bacso, S., \& Winzen, O. (2009). Technical Note: Occlusal Fingerprint Analysis: Quantification of Tooth Wear Pattern. American Journal of Physical Anthropology, 139(4), 600-605. https://doi.org/10.1002/ajpa.21086

Le Luyer, M., Coquerelle, M., Rottier, S., \& Bayle, P. (2016). Internal Tooth Structure and Burial Practices: Insights into the Neolithic Necropolis of Gurgy (France, 5100-4000 cal. BC). Plos One, 11(7), e0159688. https://doi.org/10.1371/journal.pone.0159688

Li, Z., Wang, S. H., Fan, R. R., Cao, G., Zhang, Y. D., \& Guo, T. (2019). Teeth category classification via seven-layer deep convolutional neural network with max pooling and global average pooling. International Journal of Imaging Systems and Technology, 29(4), 577-583. https://doi.org/10.1002/ima.22337

Liao, W., Xing, S., Li, D. W., Martinon-Torres, M., Wu, X. J., Soligo, C., . . Liu, W. (2019). Mosaic dental morphology in a terminal Pleistocene hominin from Dushan Cave in southern China. Scientific Reports, 9, 2347. https://doi.org/10.1038/s41598-019-38818-x

Lipton, Z. C. (2018). The Mythos of Model Interpretability. Communications of the Acm, 61(10), 36-43. https://doi.org/10.1145/3233231

Litjens, G., Kooi, T., Bejnordi, B. E., Setio, A. A. A., Ciompi, F., Ghafoorian, M., . . Sanchez, C. I. (2017). A survey on deep learning in medical image analysis. Medical Image Analysis, 42, 60-88. https://doi.org/10.1016/j.media.2017.07.005

Lockey, A. L., Alemseged, Z., Hublin, J. J., \& Skinner, M. M. (2020). Maxillary molar enamel thickness of Plio-Pleistocene hominins. Journal of Human Evolution, 142, 102731. https://doi.org/10.1016/j.jhevol.2019.102731

Martin, L. B. (1985). Significance of enamel thickness in hominoid evolution. Nature, 314(6008), 260-263. https://doi.org/10.1038/314260a0

Martin, R. M. G., Hublin, J. J., Gunz, P., \& Skinner, M. M. (2017). The morphology of the enamel dentine junction in Neanderthal molars: Gross morphology, non-metric traits, and temporal trends. Journal of Human Evolution, 103, 20-44. https://doi.org/10.1016/j.jhevol.2016.12.004

Martinón-Torres, M., de Castro, J. M. B., Gomez-Robles, A., Prado-Simon, L., \& Arsuaga, J. L. (2012). Morphological description and comparison of the dental remains from AtapuercaSima de los Huesos site (Spain). Journal of Human Evolution, 62(1), 7-58. https://doi.org/10.1016/j.jhevol.2011.08.007

Morphosource database (2020). Retrieved from https://www.morphosource.org

MorphoMuseuM database (2020). Retrieved from https://morphomuseum.com. https://doi.org/10.18563/m3.2.1.e1

Muhazid, A.A.M., Wan, W., \& Hou, L. (2020). A new volumetric CNN for 3D object classification based on joint multiscale feature and subvolume supervised learning approaches. Computational Intelligence and Neuroscience, 2020, 5851465. https://doi.org/10.1155/2020/5851465

NESPOS database (2020). NEanderthal Studies Professional Online Service. Retrieved from 
http://www.nespos.org

Olejniczak, A. J., Smith, T. M., Feeney, R. N. M., Macchiarelli, R., Mazurier, A., Bondioli, L., . . Hublin, J. J. (2008a). Dental tissue proportions and enamel thickness in Neandertal and modern human molars. Journal of Human Evolution, 55(1), 12-23. https://doi.org/10.1016/j.jhevol.2007.11.004

Olejniczak, A. J., Smith, T. M., Wang, W., Potts, R., Ciochon, R., Kullmer, O., . . Hublin, J. J. (2008b). Molar enamel thickness and dentine horn height in Gigantopithecus blacki. American Journal of Physical Anthropology, 135(1), 85-91. https://doi.org/10.1002/ajpa.20711

Olejniczak, A. J., Tafforeau, P., Feeney, R. N. M., \& Martin, L. B. (2008c). Three-dimensional primate molar enamel thickness. Journal of Human Evolution, 54(2), 187-195. https://doi.org/10.1016/j.jhevol.2007.09.014

Ortiz, A., Bailey, S. E., Delgado, M., Zanolli, C., Demeter, F., Bacon, A. M., . . Skinner, M. M. (2019). A distinguishing feature of Pongo upper molars and its implications for the taxonomic identification of isolated hominid teeth from the Pleistocene of Asia. American Journal of Physical Anthropology, 170(4), 595-612. https://doi.org/10.1002/ajpa.23928

Pope, G. G. (1983). Evidence on the age of the Asian Hominidae. Proceedings of the National Academy of Sciences of the United States of America-Biological Sciences, 80(16), 49884992. https://doi.org/10.1073/pnas.80.16.4988

Radovic, P., Lindal, J., Mihailovic, D., \& Roksandic, M. (2019). The first Neanderthal specimen from Serbia: Maxillary first molar from the Late Pleistocene of Pesturina Cave. Journal of Human Evolution, 131, 139-151. https://doi.org/10.1016/j.jhevol.2019.03.018

Shin, H. C., Roth, H. R., Gao, M. C., Lu, L., Xu, Z. Y., Nogues, I., . . Summers, R. M. (2016). Deep Convolutional Neural Networks for Computer-Aided Detection: CNN Architectures, Dataset Characteristics and Transfer Learning. IEEE Transactions on Medical Imaging, 35(5), 1285-1298. https://doi.org/10.1109/tmi.2016.2528162

Simons, E. L. (1963). Some fallacies in the study of hominid phylogeny. Science, 141(3584), 879889. https://doi.org/10.1126/science.141.3584.879

Skinner, M. M., Evans, A., Smith, T. M., Jernvall, J., Tafforeau, P., Kupczik, K.,... Hublin, J. J. (2010). Brief Communication: Contributions of enamel-dentine junction shape and enamel deposition to primate molar crown complexity. American Journal of Physical Anthropology, 142(1), 157-163. https://doi.org/10.1002/ajpa.21248

Skinner, M. M., Gunz, P., Wood, B. A., Boesch, C., \& Hublin J. J. (2009). Discrimination of extant Pan species and subspecies using the enamel-dentine junction morphology of lower molars. American Journal of Physical Anthropology, 140(2), 234-243. https://doi.org/10.1002/ajpa.21057

Skinner, M. M., Wood, B. A., Boesch, C., Olejniczak, A. J., Rosas, A., Smith, T. M., \& Hublin, J. J. (2008). Dental trait expression at the enamel-dentine junction of lower molars in extant and fossil hominoids. Journal of Human Evolution, 54(2), 173-186. https://doi.org/10.1016/j.jhevol.2007.09.012

Smith, T. M. (2016). Dental development in living and fossil orangutans. Journal of Human Evolution, 94, 92-105. https://doi.org/10.1016/j.jhevol.2016.02.008

Smith, T. M., Bacon, A. M., Demeter, F., Kullmer, O., Nguyen, K. T., Vos, J. D., . . Zhao, L. X. (2011). Dental tissue proportions in fossil orangutans from mainland Asia and Indonesia. Human Origins Research, 1, e1. https://doi.org/10.4081/hor.2011.3

Smith, T. M., Harvati, K., Olejniczak, A. J., Reid, D. J., Hublin, J. J., \& Panagopoulou, E. (2009b). Brief Communication: Dental Development and Enamel Thickness in the Lakonis 
Neanderthal Molar. American Journal of Physical Anthropology, 138(1), 112-118. https://doi.org/10.1002/ajpa.20898

Smith, T. M., Houssaye, A., Kullmer, O., Le Cabec, A., Olejniczak, A. J., Schrenk, F., .. . Tafforeau, P. (2018). Disentangling isolated dental remains of Asian Pleistocene hominins and pongines. Plos One, 13(11), e0204737. https://doi.org/10.1371/journal.pone.0204737

Smith, T. M., Olejniczak, A. J., Kupczik, K., Lazzari, V., De, V. J., Kullmer, O., . . Tafforeau, P. (2009a). Taxonomic assessment of the Trinil molars using non-destructive 3D structural and development analysis. PaleoAnthropology, 2009, 117-129.

Smith, T. M., Olejniczak, A. J., Reid, D. J., Ferrell, R. J., \& Hublin, J. J. (2006). Modern human molar enamel thickness and enamel-dentine junction shape. Archives of Oral Biology, 51(11), 974-995. https://doi.org/10.1016/j.archoralbio.2006.04.012

Smith, T. M., Tafforeauc, P., Reid, D. J., Pouech, J., Lazzari, V., Zermeno, J. P., . . . Hublin, J. J. (2010). Dental evidence for ontogenetic differences between modern humans and Neanderthals. Proceedings of the National Academy of Sciences of the United States of America, 107(49), 20923-20928. https://doi.org/10.1073/pnas.1010906107

Tafforeau, P. (2004). Phylogenetic and functional aspects of tooth enamel microstructure and threedimensional structure of modern and fossil primate molars. (Ph. D. Dissertation), Universite de Montpellier II, Languedoc Roussilon, France.

Tshen, L. T. (2016). Biogeographic distribution and metric dental variation of fossil and living orangutans (Pongo spp.). Primates, 57(1), 39-50. https://doi.org/10.1007/s10329-0150493-z

von Koenigswald, G. H. R. (1957). Remarks on Gigantopithecus and other hominid remains from South China. Proc. Roy. Acad. Amsterdam Ser. B, 60, 153-159.

Wang, C. B., Zhao, L. X., Jin, C. Z., Wang, Y., Qin, D. G., \& Pan, W. S. (2014). New discovery of Early Pleistocene orangutan fossils from Sanhe Cave in Chongzuo, Guangxi, southern China. Quaternary International, 354, 68-74. https://doi.org/10.1016/j.quaint.2014.06.020

Wang, W. (2009). New discoveries of Gigantopithecus blacki teeth from Chuifeng Cave in the Bubing Basin, Guangxi, south China. Journal of Human Evolution, 57(3), 229-240. https://doi.org/10.1016/j.jhevol.2009.05.004

Wang, W., Potts, R., Baoyin, Y., Huang, W. W., Cheng, H., Edwards, R. L., \& Ditchfield, P. (2007). Sequence of mammalian fossils, including hominoid teeth, from the Bubing Basin caves, South China. Journal of Human Evolution, 52(4), 370-379. https://doi.org/10.1016/j.jhevol.2006.10.003

Wolpoff, M. H. (1982). Ramapithecus and hominid origins. Current Anthropology, 23, 501-522. https://doi.org/10.1086/202893

Xu, X. J., Liu, C., \& Zheng, Y. Y. (2019). 3D Tooth Segmentation and Labeling Using Deep Convolutional Neural Networks. Ieee Transactions on Visualization and Computer Graphics, 25(7), 2336-2348. https://doi.org/10.1109/tvcg.2018.2839685

Yao, Y. Y., Liao, W., Bae, C. J., Sun, X. F., Feng, Y. X., Tian, C., . . . Wang, W. (2020). New discovery of Late Pleistocene modern human teeth from Chongzuo, Guangxi, southern China. Quaternary International, 563, 5-12. https://doi.org/10.1016/j.quaint.2020.02.002

Zanolli, C., Bondioli, L., Mancini, L., Mazurier, A., Widianto, H., \& Macchiarelli, R. (2012). Brief communication: Two human fossil deciduous molars from the sangiran dome (Java, Indonesia): Outer and inner morphology. American Journal of Physical Anthropology, 147(3), 472-481. https://doi.org/10.1002/ajpa.21657

Zanolli, C., Grine, F. E., Kullmer, O., Schrenk, F., \& Macchiarelli, R. (2015). The early Pleistocene deciduous hominid molar FS-72 from the Sangiran dome of java, Indonesia: A taxonomic 
reappraisal based on its comparative endostructural characterization. American Journal of Physical Anthropology, 157(4), 666-674. https://doi.org/10.1002/ajpa.22748

Zanolli, C., Kullmer, O., Kelley, J., Bacon, A. M., Demeter, F., Dumoncel, J., . . Macchiarelli, R. (2019). Evidence for increased hominid diversity in the Early to Middle Pleistocene of Indonesia. Nature Ecology \& Evolution, 3(5), 755-764. https://doi.org/10.1038/s41559019-0860-Z

Zhang, Q. S., \& Zhu, S. C. (2018). Visual interpretability for deep learning: a survey. Frontiers of Information Technology \& Electronic Engineering, 19(1), 27-39. https://doi.org/10.1631/fitee.1700808

Zhang, Y. Q., \& Harrison, T. (2017). Gigantopithecus blacki: a giant ape from the Pleistocene of Asia revisited. American Journal of Physical Anthropology, 162, 153-177. https://doi.org/10.1002/ajpa.23150 